\title{
Nîmes
}

Rue de l'Agau

\section{Martial Monteil}

\section{(2) OpenEdition}

1 Journals

Édition électronique

URL : http://journals.openedition.org/adlfi/11720

ISSN : 2114-0502

Éditeur

Ministère de la culture

Référence électronique

Martial Monteil, « Nîmes », ADLFI. Archéologie de la France - Informations [En ligne], Languedoc-

Roussillon, mis en ligne le 01 mars 2004, consulté le 03 mai 2019. URL : http://

journals.openedition.org/adlfi/11720

Ce document a été généré automatiquement le 3 mai 2019.

(c) Ministère de la Culture et de la Communication, CNRS 


\title{
Nîmes
}

\author{
Rue de l'Agau
}

Martial Monteil

Date de l'opération : 1994 (SD) ; 1994 (SU)

Inventeur(s) : Monteil Martial (AFAN)

1 Plan général des opérations intra muros de Nîmes (Fig. $n^{\circ} 3$ : En noir, les principales fouilles réalisées entre 1965 et 1990 à l'intérieur de la ville du Haut-Empire, associées à quelques grands repères topographiques).

2 La fouille a été engagée préalablement à la réalisation d'une résidence pour étudiants et d'une salle polyvalente (maison du protestantisme), au 67, rue de l'Agau - 3, rue ClaudeBrousson (DO-1369, DO-1313). Les parcelles concernées se situent en bordure de la rue de l'Agau, prolongement de la rue Nationale : cet axe actuel recouvre peu ou prou le tracé de la voie domitienne, transformé, avec la progression de l'urbanisme vers l'ouest, en rue majeure de la ville antique.

3 Les tranchées de repérage et la fouille ont permis de dégager une surface totale d'environ $150 \mathrm{~m}^{2}$, qui se réduit à moins d'une dizaine de mètres carrés pour les horizons les plus profonds [ (Fig. $n^{\circ} 1$ : Plan cumulé des vestiges de la rue de l'Agau, avec indication des zones de fouille) ; (Fig. n 2 : Rue de l'Agau. Plans de détail de trois des principales phases d'occupation perceptibles. A : dans le courant du $\mathrm{I}^{\mathrm{er}}$ s. av. J.-C. ; B : autour du changement d'ère ; $\mathrm{C}$ : dans la seconde moitié du $\mathrm{II}^{\mathrm{e}} \mathrm{s}$. apr. J.-C.)]. Au terme de cette opération, plusieurs contraintes ont incité les aménageurs à choisir une construction sur dalle flottante, après exhaussement du terrain : les vestiges mis en évidence ont donc été préservés [(Fiches, Jean-Luc (dir.) ; Veyrac, Alain (dir.). 1996.) ; (Monteil, Martial. 1999.)].

\section{Premières traces d'occupation}

4 La profondeur importante du sol géologique dans ce secteur, supérieure à $4 \mathrm{~m}$ sous le terrain actuel, ainsi que l'observation, dans un étroit sondage, de dépôts liés au passage de l'eau, permettent de restituer une dépression naturelle. Reliée à d'autres observations 
voisines, cette information incite à reconnaître ici le passage de l'effluent de la source de la Fontaine, évoqué par ailleurs dans la notice consacrée au site du boulevard Gambetta (Monteil, Martial. 1999.). Dans le courant du I ${ }^{\mathrm{er}}$ s. av. J.-C., un canal, d'une largeur supérieure à 1,80 m se surimpose au cours d'eau colmaté. Seuls ont été observés un de ses parements et son dallage de fond.

\section{Un canal d'époque augustéenne}

5 Aux alentours du changement d'ère, cet aménagement hydraulique est en partie détruit par une autre canalisation plus monumentale ( $\mathrm{CN} 43)$. Celle-ci, qui suit la même orientation est-ouest, peut être restituée sur $18 \mathrm{~m}$ de long. Son canal, large de 1,85 m à $2 \mathrm{~m}$, est limité par des murs dont les parements intérieurs sont construits avec soin : une assise de blocs de grand appareil supportant des assises régulières de moellons bien taillés et liés au mortier. Le fond, pavé de dalles calcaires, permet de donner une hauteur totale de $1,50 \mathrm{~m}$ à ce canal à ciel ouvert. Son rôle initial est probablement de canaliser une eau vive qu'il serait tentant de voir issue de la source de la Fontaine. En parallèle, ses abords et, sans doute plus globalement, l'ensemble du secteur sont exhaussés.

6 Très vite pourtant, entre le changement d'ère et le premier quart du $\mathrm{I}^{\mathrm{er}} \mathrm{s}$. apr. J.-C., le fond du canal est remblayé par un dépotoir sur environ $0,60 \mathrm{~m}$ d'épaisseur. Au même moment, ou peu après, les terrains alentour sont à nouveau exhaussés d'environ $1 \mathrm{~m}$ et les parements du canal surélevés d'autant. Ce réaménagement s'explique sans doute par des problèmes liés à la circulation des eaux.

7 À compter du premier quart du $\mathrm{I}^{\mathrm{er}}$ s. apr. J.-C., ce canal est longé par des maisons et sert désormais aussi d'égout collecteur des eaux usées. Il constitue une limite forte, qui subsiste jusqu'à l'abandon définitif du site, et l'aplomb surélevé de ses murs latéraux sert, en outre, de façade aux maisons mitoyennes. Durant le $\mathrm{I}^{\text {er }}$ s. apr. J.-C. et le II ${ }^{\mathrm{e}}$ s. apr. J.-C., il sert ponctuellement de lieu de décharge dans lequel s'accumulent graduellement des déchets domestiques. L'épaisseur de dépôts reste cependant relativement modérée et ne semble pas nuire à l'écoulement des eaux.

\section{Au sud du canal}

8 Côté ouest, une tranchée ponctuelle a mis au jour une portion de sol en terrazzo blanc (SL25). Au sud-est, une pièce, reconnue sur une petite surface, est définie par un sol de mosaïque blanche (SL10). Elle jouxte une probable galerie, large de 2,80 m et orientée estouest, qui comporte un sol en béton de tuileau (SL12). Contre le canal, trois espaces peuvent être distingués : une première pièce, d'une largeur comprise entre 2,50 $\mathrm{m}$ et $3 \mathrm{~m}$, et pourvue d'un sol en terrazzo blanc décoré de lignes de croisettes (SL14); un probable passage d'échelle (limite de propriété), large de $0,90 \mathrm{~m}$, matérialisé par un petit égout (CN66); une seconde pièce incomplète avec sol en terrazzo décoré d'une résille d'hexagones en tesselles noires.

9 Les seules restructurations perceptibles au sein de ces six espaces, mis à part les traces laissées par l'abandon des lieux, sont celles marquées par la réfection du sol SL12 de la galerie et par le remblaiement du probable passage d'échelle. Ce dernier est rehaussé, dans le courant de la seconde moitié du $\mathrm{II}^{\mathrm{e}} \mathrm{s}$. apr. J.-C., et supporte un nouveau sol de circulation en terre battue plus proche de la surface topographique des pièces voisines. 


\section{Au nord du canal} terre battue signalée précédemment est pourvue d'un pavement en terrazzo blanc décoré, dans sa partie centrale, de crustae et de croisettes de tesselles noires (SL5). Elle est flanquée, dans l'angle sud-est, par un couloir à sol en terre battue, dont trois états ont pu être reconnus. Vers l'est, une cour est bordée, côté canal, par deux fondations, probables supports d'une colonnade. Enfin, à l'extrémité est de cette cour, un réseau quasi inextricable de murs très épierrés ou partiellement conservés a pu être repéré, en liaison avec une portion congrue d'une mosaïque à gros cubes blancs (SL 24).

L'ensemble de ces espaces est apparemment construit dans le courant des deuxième et troisième quarts du $\mathrm{I}^{\mathrm{er}} \mathrm{s}$. apr. J.-C., mais ils trouvent sans doute une partie de leur origine dans les aménagements initiaux du début du $\mathrm{I}^{\mathrm{er}} \mathrm{s}$. Seule la cour témoigne d'un second état tangible, daté de la seconde moitié du II ${ }^{\mathrm{e}} \mathrm{s}$. apr. J.-C. À cette époque, le parement nord du canal est partiellement détruit et remplacé par un mur légèrement biaisé qui constitue donc une nouvelle façade. Les sols de terre battue de cette cour couvrent une période comprise entre le premier quart du $\mathrm{I}^{\mathrm{er}} \mathrm{s}$. apr. J.-C. et le début du III ${ }^{\mathrm{e}}$ s. apr. J.-C.

Plus au nord, un puissant mur, large de $0,89 \mathrm{~m}$, pourrait correspondre à une limite de la rue antique traditionnellement restituée dans l'axe de la rue de l'Agau actuelle. Ce mur est parallèle à une portion de seuil à glissière qui sépare deux sols de terre battue, datés de la première moitié du III $\mathrm{e}$. apr. J.-C.

\section{Le site durant l'Antiquité tardive}

Quelques éléments ténus marquent une transformation tardive de la maison située sur la rive gauche du canal. Vers la fin du IV $\mathrm{e}$ s. apr. J.-C. ou dans la première moitié du V $\mathrm{V}^{\mathrm{e}}$ s. apr. J.-C., une chape de mortier beige recouvre une partie de la cour et de la pièce au sol décoré de crustae. À la même période, le canal est remblayé jusqu'au contact de son arase initiale. Une unique dalle monolithe en calcaire peut suggérer qu'un dallage est alors mis en place pour permettre la continuité de l'évacuation des eaux. Quoi qu'il en soit, l'écoulement d'eau persiste un temps, puisque les remblais tardifs sont recouverts par une sédimentation d'origine hydraulique. Peu de temps après, mais dans le même intervalle chronologique, les murs des maisons sont épierrés et le canal définitivement condamné. Une fosse tardive, établie dans l'ancienne cour, a livré le mobilier le plus tardif, datable de la seconde moitié du Ve s., voire du début du VI ${ }^{e}$ s. apr. J.-C. Enfin, audessus de l'ensemble de ces vestiges, une couche souvent épaisse de plus d'un mètre paraît marquer une reprise de la pédogenèse. Le peu de mobilier qu'elle inclut date du bas Moyen Âge et de l'époque moderne, mais sa formation a pu débuter antérieurement. 


\section{BIBLIOGRAPHIE}

Fiches, Jean-Luc (dir.)Veyrac, Alain (dir.). 1996 : Nîmes, Paris, Académie des inscriptions et BellesLettres, coll. « Carte archéologique de la Gaule », 30-1, Pré-inventaire archéologique, 634 p., 31 p. de $\mathrm{pl}$.

Monteil, Martial. 1999 : Nîmes antique et sa proche campagne, Étude de topographie urbaine et périurbaine (fin VIe s. av. J.-C. - VIe s. apr. J.-C.), Lattes (coll. Monographies d'archéologie méditerranéenne, 3), $528 \mathrm{p}$.

Monteil, Martial. 1999 : Nîmes antique et sa proche campagne, Étude de topographie urbaine et périurbaine (fin VIe s. av. J.-C. - VIe s. apr. J.-C.), Lattes (coll. Monographies d'archéologie méditerranéenne, 3), $528 \mathrm{p}$.

\section{ANNEXES}

Fig. $n^{\circ} 1$ : Plan cumulé des vestiges de la rue de l'Agau, avec indication des zones de fouille

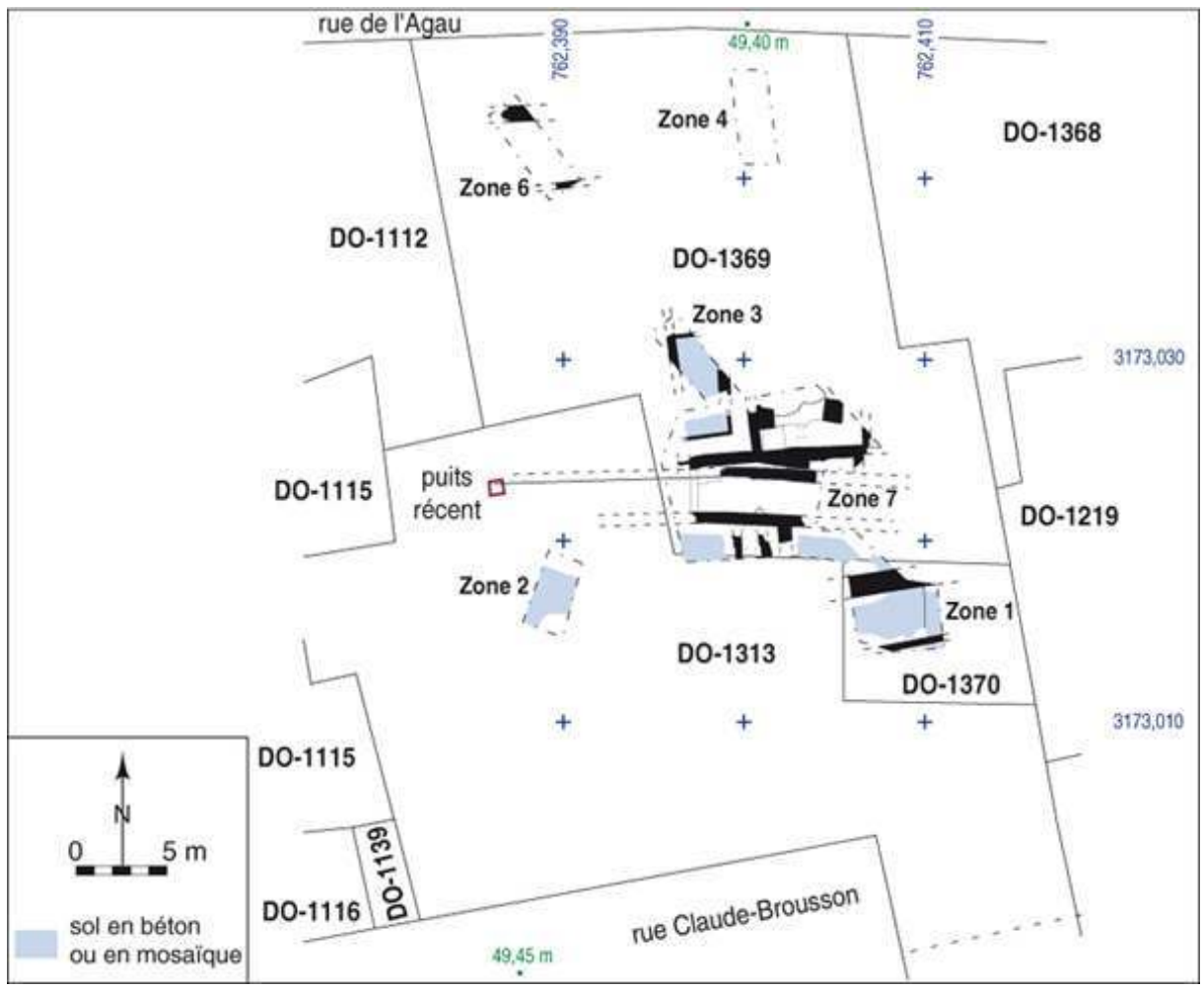

Auteur(s) : Monteil, Martial. Crédits : ADLFI - Monteil, Martial (2004) 
Fig. $n^{\circ} 2$ : Rue de l'Agau. Plans de détail de trois des principales phases d'occupation perceptibles. A : dans le courant du $\mathrm{I}^{\mathrm{er}} \mathrm{S}$. av. J.-C. ; B : autour du changement d'ère ; C : dans la seconde moitié du II s. apr. J.-C.

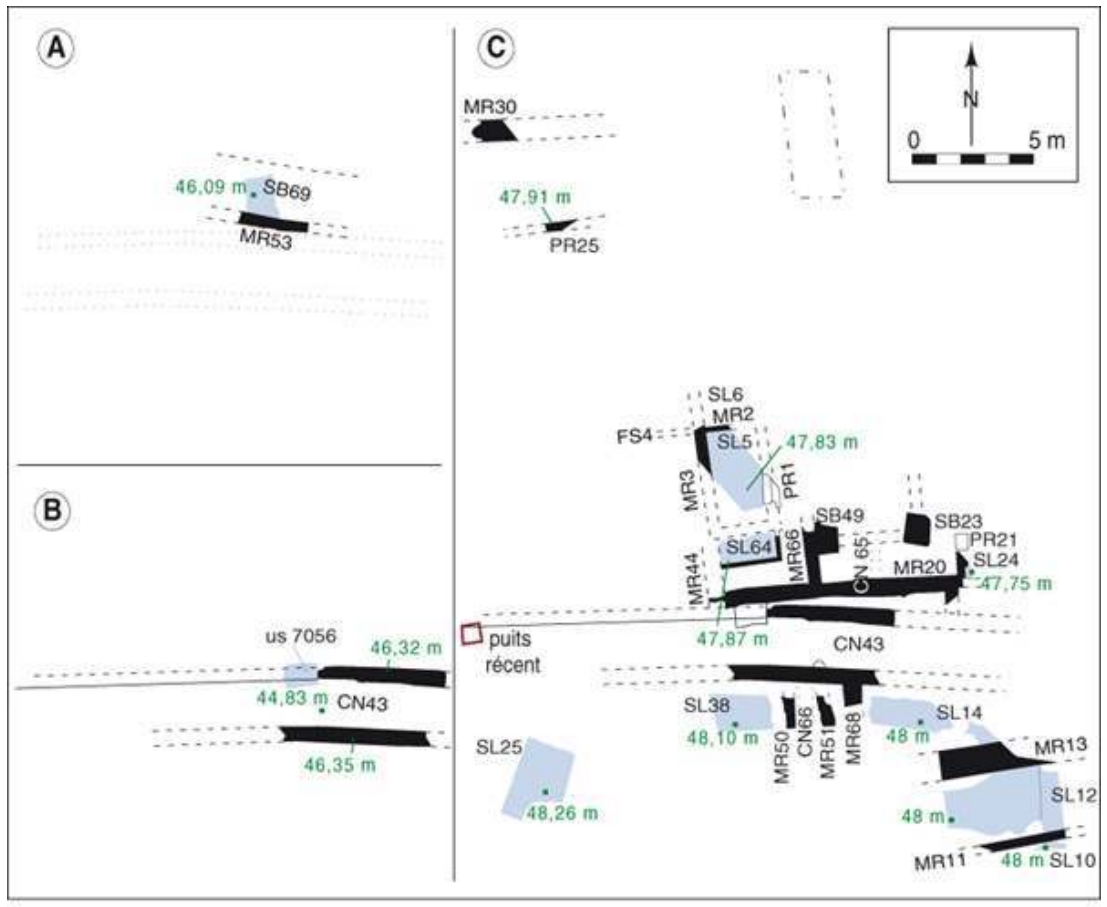

Auteur(s) : Monteil, Martial. Crédits : ADLFI - Monteil, Martial (2004) 
Fig. $n^{\circ} 3$ : En noir, les principales fouilles réalisées entre 1965 et 1990 à l'intérieur de la ville du Haut-Empire, associées à quelques grands repères topographiques

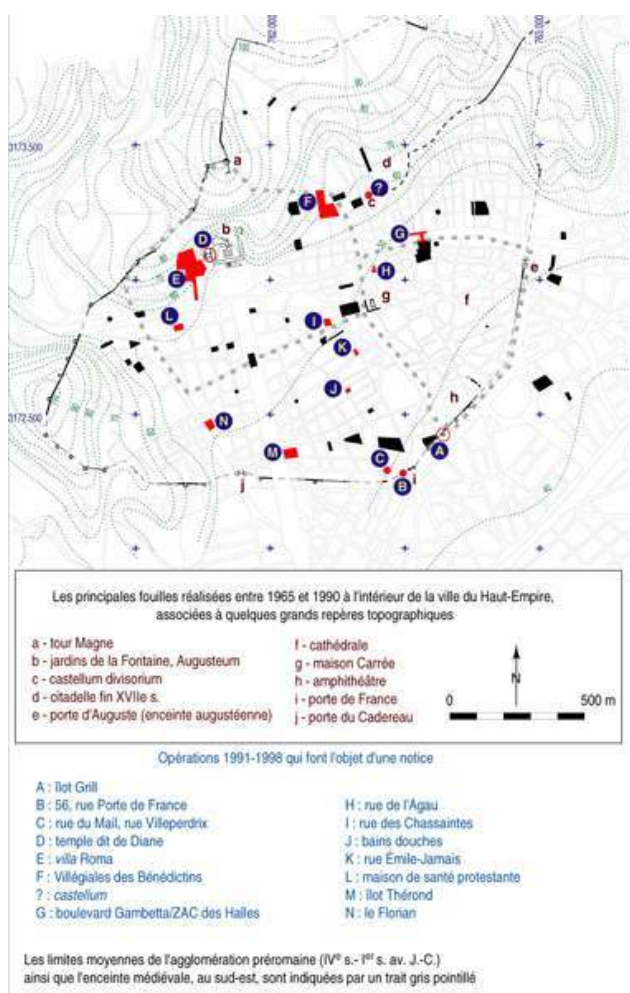

Auteur(s) : Monteil, Martial. Crédits : ADLFI - Monteil, Martial (2004)

INDEX

Index géographique : Languedoc-Roussillon, Gard (30), Nîmes Index chronologique : République romaine, Haut-Empire operation Sondage (SD), Sauvetage urgent (SU)

\section{AUTEURS}

MARTIAL MONTEIL

AFAN 